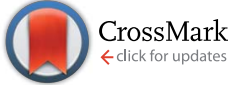

Cite this: RSC Adv., 2016, 6, 57103

Received 29th February 2016 Accepted 28th May 2016

DOI: $10.1039 / c 6 r a 05405 f$

\section{Production and electrical characterization of the reflectin A2 isoform from Doryteuthis (Loligo) pealeii†}

\author{
David D. Ordinario, $\S^{\mathrm{a}}$ Long Phan, $\S^{\mathrm{a}}$ Ward G. Walkup IV, $\$ \S^{\mathrm{a}}$ Yegor Van Dyke, ${ }^{\mathrm{a}}$ \\ Erica M. Leung, ${ }^{a}$ Michael Nguyen, ${ }^{a}$ Amanda G. Smith, ${ }^{a}$ Justin Kerr, ${ }^{a}$ Mahan Naeim, ${ }^{a}$
} Ioannis Kymissis ${ }^{\mathrm{b}}$ and Alon A. Gorodetsky ${ }^{\star a c}$

www.rsc.org/advances

Cephalopods have recently emerged as a source of inspiration for the development of novel functional materials. Within this context, a number of studies have explored structural proteins known as reflectins, which play a key role in cephalopod adaptive coloration in vivo and exhibit interesting properties in vitro. Herein, we report an improved high-yield strategy for the preparation and isolation of reflectins in quantities sufficient for materials applications. We first select the Doryteuthis (Loligo) pealeii reflectin A2 (RfA2) isoform as a "model" system and validate our approach for the expression and purification of this protein. We in turn fabricate RfA2-based twoterminal devices and employ both direct and alternating current measurements to demonstrate that RfA2 films conduct protons. Our findings underscore the potential of reflectins as functional materials and may allow a wider range of researchers to investigate their properties.

Cephalopods (squid, octopuses, and cuttlefish) are well known for their sophisticated neurophysiology, complex behavior, and stunning camouflage displays. ${ }^{\mathbf{1 - 6}}$ Recently, these animals have drawn significant attention as sources of novel materials for optical systems, ${ }^{7-10}$ biomedical technologies, ${ }^{11-15}$ and bioelectronic devices. ${ }^{\mathbf{1 6 - 2 0}}$ Within this context, a number of literature reports have investigated the properties of unique structural proteins known as reflectins, ${ }^{7-10,14,16-18,21-24}$ which are found in cephalopod skin cells (i.e. leucophores, iridophores, and chromatophores). ${ }^{25-30}$ In vivo, reflectins in general have been shown to play important roles in cephalopod adaptive coloration by serving as components of optically-active

${ }^{a}$ Department of Chemical Engineering and Materials Science, University of California, Irvine, CA 92697, USA. E-mail: alon.gorodetsky@uci.edu

${ }^{b}$ Department of Electrical Engineering, Columbia University, New York, NY 10027, USA

${ }^{c}$ Department of Chemistry, University of California, Irvine, CA 92697, USA

$\dagger$ Electronic supplementary information (ESI) available. See DOI: 10.1039/c6ra05405f

$\S$ Denotes equal contribution.

\$ Current address: Department of Biology and Biological Engineering, California Institute of Technology, Pasadena, CA 91125, USA. ultrastructures, including layered stacks of membrane-enclosed platelets in iridophores, ${ }^{25,26}$ membrane-bound arrangements of spherical microparticles in leucophores, ${ }^{27,28}$ and interconnected networks of pigment granules in chromatophores. ${ }^{29,30}$ In vitro, the Doryteuthis (Loligo) pealeii reflectin A1 (RfA1) isoform has found applications in reconfigurable infrared camouflage coatings that are actuated by chemical and mechanical stimuli, ${ }^{7,8}$ proton-conducting films with electrical figures of merit rivaling those of some artificial analogues, ${ }^{\mathbf{1 6 - 1 8}}$ and biocompatible substrates that support the proliferation and differentiation of neural stem cells. ${ }^{14}$ Overall, reflectins' fascinating properties have provided a strong impetus for their continued exploration from both fundamental and applied perspectives.

Herein, we describe an improved methodology for the production of difficult-to-handle reflectins in quantities sufficient for materials applications. We first select the Doryteuthis (Loligo) pealeii reflectin A2 (RfA2) isoform as a "model" system for electrical characterization and validate a new high-yield strategy for the expression and purification of this precipitation-prone protein. We subsequently fabricate and characterize two-terminal devices for which RfA2 thin films constitute the active layer. We in turn interrogate RfA2-based devices featuring palladium and palladium hydride electrodes via direct current electrical measurements and RfA2-based devices featuring gold electrodes via alternating current electrical measurements. From our experiments, we glean that RfA2 is an effective proton-conducting material, with properties similar to those of RfA1. Overall, our findings underscore the potential of reflectins as functional materials and may allow a wider range of researchers to investigate their properties.

Having previously demonstrated that Doryteuthis (Loligo) pealeii reflectin $\mathrm{A} 1$ is an excellent proton conductor, ${ }^{\mathbf{1 6 - 1 8}}$ we sought to extend these studies and explore the electrical functionality of other reflectins. For our experiments, we specifically selected Doryteuthis (Loligo) pealeii reflectin A2 (Fig. 1A) due to its key biological role in the dynamic optical functionality of iridophores ${ }^{23,26}$ and its similarity to reflectins from other squid 
A)

MDXXXXXDXXXX

1 MNRYMMRHRPMYSNMYRTGRKYRGVMEPMSRMTMDFQGRYMDSQGRMVDPRYYDYGRCHD

60

MDXXXXXMXXXXXMDXXXXXMDXXXX

61

YDRYYGRSMFNYGPNMDGQRYGGWMDEPERYMDMSGYQMDMHGRWMDSQGRYCNPMGHSW

MDXXXXXMDXXXXXMDXX:

121

SNRQGYYPGSNYGRNMFNPERYMDMSGYQMDMQGRWMDMGGRHVNPFSHSMYGRNMFNPS

MDXXXXMDXXXXMDXXXXMDXXXXMXXX8

181

YFSNRHMDNPERYMDMSGYQMDMQGRWMDTQGRYMDPSWSNMYDNYNSWY
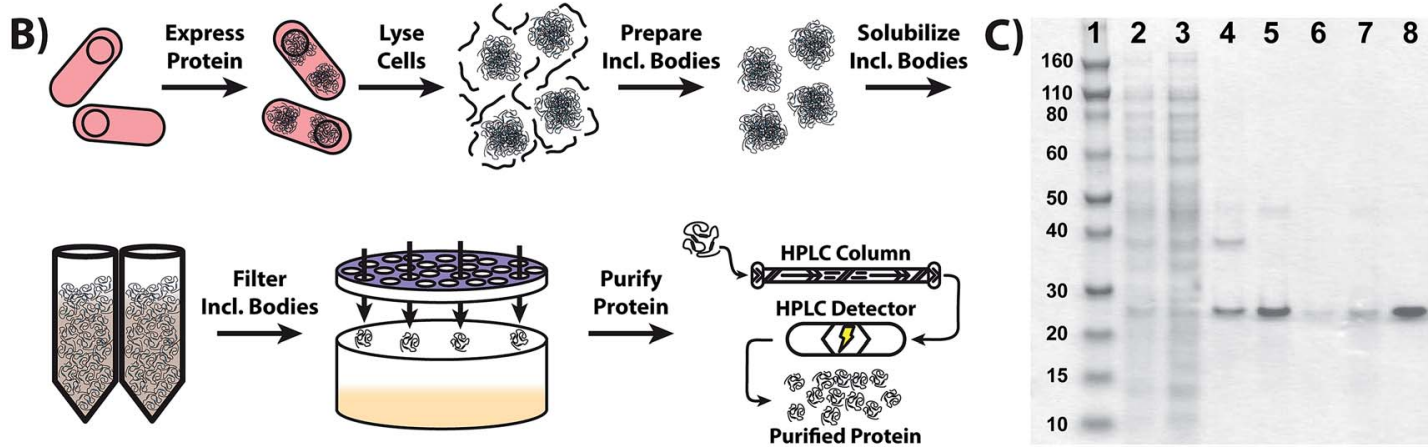

Fig. 1 (A) Illustration of the primary sequence of Doryteuthis (Loligo) pealeii reflectin A2. The conserved sequence motifs of the form (M/F-D$\left.X_{5}\right)\left(M-D-X_{5}\right)_{n}\left(M-D-X_{3 / 4}\right)$ are indicated above the sequence in light blue. The conserved aspartic acid and methionine residues present in the subdomains are indicated in bolded red and bolded black, respectively. (B) Illustration of the expression and purification of RfA2. The procedure entails protein expression, cell lysis, inclusion body preparation, inclusion body solubilization, inclusion body filtration, and protein purification via high performance liquid chromatography. (C) Analysis of the expression and purification of RfA2 via sodium dodecyl sulfate polyacrylamide gel electrophoresis (SDS-PAGE). The stain indicates the total protein. The individual lanes correspond to: lane 1, 10-160 kDa molecular weight standards; lane 2, total protein; lane 3, soluble protein; lane 4, insoluble protein; lane 5, solubilized inclusion bodies; lane 6, filtered inclusion bodies; lane 7, concentrated filtered inclusion bodies; lane 8, HPLC eluate.

species (with the exception of reflectin B1). Indeed, RfA2 from $D$. pealeii features a $>60 \%$ pair-wise amino acid sequence identity with the majority of isolated reflectins from E. scolopes, ${ }^{21} D$. opalescens ${ }^{26}$ and $L$. forbesi, ${ }^{31}$ and much like its homologues, this reflectin variant contains multiple characteristic $(\mathrm{M} / \mathrm{F}-\mathrm{D}-$ $\left.\mathrm{X}_{5}\right)\left(\mathrm{M}-\mathrm{D}-\mathrm{X}_{5}\right)_{n}\left(\mathrm{M}-\mathrm{D}-\mathrm{X}_{3 / 4}\right)$ sequence motifs and large percentages of both aromatic and charged amino acids (Fig. 1A). ${ }^{23,26}$ Furthermore, although the biochemical characterization of RfA2 has been reported, ${ }^{23,24,26}$ the protein has not been studied as a functional material to date and possesses completely unexplored electrical properties. Given the aforementioned considerations, we viewed RfA2 as a judicious "model" reflectin for electrical characterization and further investigation.

We began our studies by developing and implementing an improved strategy for the production of RfA2 in high yield (Fig. 1B), with each step of the revised procedure monitored via gel electrophoresis (Fig. 1C). First, we used protocols validated for RfA1 to heterologously express histidine-tagged RfA2 in $E$. coli, ${ }^{7,16}$ finding that the protein was sequestered within intracellular inclusion bodies (as reported for RfA1). The inclusion bodies were then prepared/isolated through several rounds of non-ionic detergent extraction and centrifugation. Subsequently, the nearly pure RfA2 (from the inclusion bodies) was solubilized through repeated manual agitation. Here, when attempting to purify the protein via immobilized metal ion affinity chromatography (IMAC), we found that even under denaturing conditions, RfA2 was prone to irreversible selfassembly into larger aggregates and/or spontaneous precipitation (as reported for some reflectins).,10,22,24 Our standard protocol ${ }^{7,16}$ thus furnished relatively small amounts of material, which were appropriate for basic characterization but not adequate for high throughput device fabrication. Consequently, rather than relying upon a time-consuming, expensive, and difficult IMAC step, we revised our procedure and simply filtered the RfA2 solutions to remove any insoluble debris and/ or precipitates. We in turn isolated the desired protein from the filtrate via high performance liquid chromatography (HPLC), obtaining typical estimated purities of $>95 \%$ (ESI Fig. S1 $\dagger$ ). Finally, we confirmed the identity of the protein via in-gel tryptic digestion and tandem mass spectrometry, obtaining typical sequence coverages of $>80 \%$ (ESI Fig. S2 $\dagger$ ). Notably, due to the use of histidine-tagged RfA2, we were able to directly 
compare the efficacy of the previously reported ${ }^{7,16}$ and current procedures, discovering that our new streamlined strategy not only avoided a challenging column chromatography step but also improved the yield by well over an order of magnitude to $>200 \mathrm{mg}$ of pure protein per liter of cell culture.

With our desired material in hand, we proceeded to fabricate two-terminal bottom contact devices according to the scheme illustrated in Fig. 2A. ${ }^{\mathbf{1 6 - 1 8}}$ First, we prepared arrayed palladium or gold metal contacts on either silicon dioxide/silicon or glass substrates, respectively, via electron beam physical vapor deposition through a shadow mask. Next, we dropcast aqueous solutions of RfA2 directly onto the electrode arrays. We then allowed the residual solvent to evaporate, prior to removing excess material through mechanical scribing. These arrayed devices enabled high throughput physical and electrical characterization of RfA2 films.

We next characterized our RfA2-based devices with optical microscopy and atomic force microscopy (AFM). ${ }^{16-18}$ The optical microscopy experiments indicated that the RfA2 active layers contained few apparent defects (representative images for palladium-contacted devices are shown in Fig. 2B and ESI Fig. S3†). The AFM experiments indicated that the RfA2 films were relatively uniform with root mean square (RMS) roughnesses of $>3 \mathrm{~nm}$ (representative images are shown in Fig. $2 \mathrm{C}$ and ESI Fig. S3†). The physical characterization experiments confirmed the integrity of our films and facilitated interpretation of the electrical measurements.

We subsequently investigated the electrical properties of RfA2 films contacted by proton-blocking palladium and protoninjecting palladium hydride electrodes (Fig. 3A). Thus, we first recorded current $(I)$ as a function of voltage $(V)$ at a relative humidity $(\mathrm{RH})$ of $80 \%$ for palladium-contacted devices (representative measurements are shown in Fig. 3B and ESI Fig. S4 $\dagger$ ). From the $I-V$ characteristics, we extracted an average current density of $7.3 \pm 4.5 \times 10^{-4} \mathrm{~A} \mathrm{~cm}^{-2}$ at $1.5 \mathrm{~V}$ across 6 independent palladium-contacted devices. We in turn formed protontransparent palladium hydride contacts via exposure of the

A)

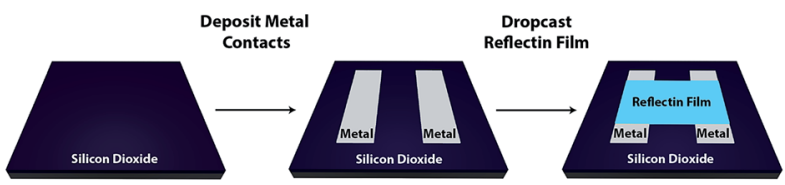

B)

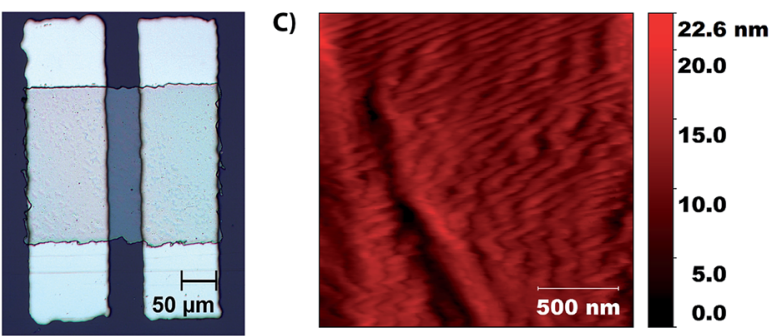

Fig. 2 (A) General scheme for the fabrication of RfA2-based devices (B) A representative optical image of a completed device for which an RfA2 film bridges two palladium electrodes. (C) A representative atomic force microscopy (AFM) image of an RfA2 film.
A)

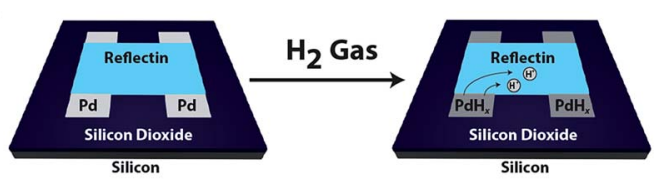

B)

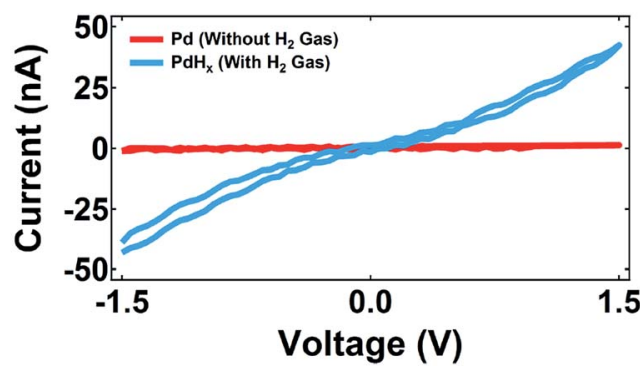

Fig. 3 (A) An illustration of an RfA2-based two-terminal device before and after in situ treatment with hydrogen $\left(\mathrm{H}_{2}\right)$ gas. The palladium $(\mathrm{Pd})$ electrodes are converted to palladium hydride $\left(\mathrm{PdH}_{x}\right)$ electrodes in the presence of $\mathrm{H}_{2}$, enabling the injection of protons into the film. (B) The current versus voltage characteristics of an RfA2 film contacted with palladium (red) and palladium hydride (blue) electrodes. The magnitude of the current increases by more than an order of magnitude upon moving from proton-blocking to proton-injecting electrodes. Both the forward and reverse scans are shown for each measurement.

palladium contacts to hydrogen gas in situ (Fig. 3A), ${ }^{\mathbf{1 6 - 2 0 , 3 2}}$ prior to again recording current as a function of voltage at an $\mathrm{RH}$ of $80 \%$ (representative measurements are shown in Fig. 3B and ESI Fig. S4 $\dagger$ ). From the $I-V$ characteristics, we extracted an average current density of $1.8 \pm 0.6 \times 10^{-2} \mathrm{~A} \mathrm{~cm}^{-2}$ at $1.5 \mathrm{~V}$ across 6 independent palladium hydride-contacted devices. Here, we found that the characteristics displayed hysteresis between the forward and reverse scans and that the observed current density increased by more than an order of magnitude upon conversion of the contacts from proton-blocking to proton-injecting (in close agreement with literature precedent for RfA1 ${ }^{16-18}$ and derivatized chitosan ${ }^{19,20}$ ). Interestingly, the current density found for RfA2 was lower than the current density of $\sim 2.6 \times 10^{-2} \mathrm{~A} \mathrm{~cm}^{-2}$ previously found for RfA1 at an $\mathrm{RH}=80 \%{ }^{16}$ This difference could be rationalized by analyzing the two proteins' histidine-tagged amino acid sequences (ESI Fig. S5 $\dagger$ ); relative to RfA1, RfA2 possesses a small but significant decrease in its percentage of charged amino acids (which are crucial for proton transport) ${ }^{\mathbf{1 6}}$ and thus might be expected to function less effectively as a proton conductor. Altogether, our observations provided strong evidence that RfA2 was a protonconducting material.

To gain additional insight into the electrical properties of RfA2, we used electrochemical impedance spectroscopy to interrogate RfA2 films contacted by gold electrodes in the presence of water $\left(\mathrm{H}_{2} \mathrm{O}\right)$ and deuterium oxide $\left(\mathrm{D}_{2} \mathrm{O}\right)$ vapor (Fig. 4A). Thus, we first recorded Nyquist plots of the imaginary versus the real parts of the impedance at an $\mathrm{RH}$ of $80 \%$ for devices in the presence of $\mathrm{H}_{2} \mathrm{O}$ (representative measurements are shown in Fig. 4B and ESI Fig. S6 $\dagger$ ). By fitting our curves with a simple equivalent circuit that accounted for the bulk impedance and capacitive effects at the electrodes, ${ }^{\mathbf{1 6}}$ we extracted an 
A)

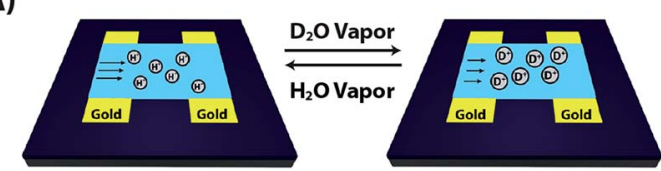

B)

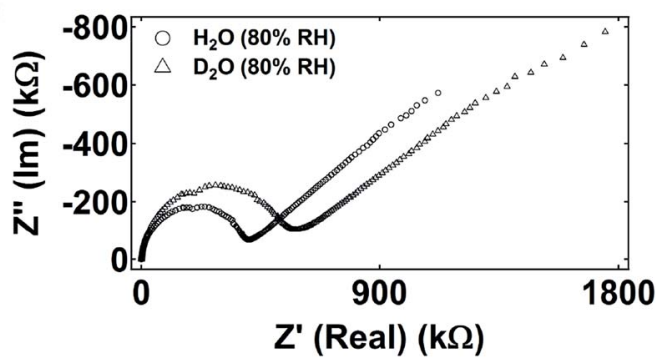

Fig. 4 (A) An illustration of a two-terminal RfA2-based device in the presence of water $\left(\mathrm{H}_{2} \mathrm{O}\right)$ vapor and deuterium oxide $\left(\mathrm{D}_{2} \mathrm{O}\right)$ vapor. (B) $A$ representative Nyquist plot of the imaginary versus the real parts of the impedance for an RfA2-based two-terminal device in the presence of $\mathrm{H}_{2} \mathrm{O}$ (open circles) and $\mathrm{D}_{2} \mathrm{O}$ (open triangles). There is a change in the effective film resistance upon moving from $\mathrm{H}_{2} \mathrm{O}$ to $\mathrm{D}_{2} \mathrm{O}$, demonstrating the kinetic isotope effect for the RfA2 film.

average conductivity of $7.4 \pm 2.0 \times 10^{-5} \mathrm{~S} \mathrm{~cm}^{-1}$ across 5 independent devices in the presence of $\mathrm{H}_{2} \mathrm{O}$. We in turn altered the environment from $\mathrm{H}_{2} \mathrm{O}$ to $\mathrm{D}_{2} \mathrm{O}$ vapor in situ (Fig. $4 \mathrm{~A}$ ), prior to again recording the imaginary versus the real parts of the impedance at an $\mathrm{RH}$ of $80 \%$ (representative measurements are shown in Fig. 4B and ESI Fig. S6 $\dagger$ ). From fits of this data, we extracted an average conductivity of $4.3 \pm 1.1 \times 10^{-5} \mathrm{~S} \mathrm{~cm}^{-1}$ across 5 independent devices in the presence of $\mathrm{D}_{2} \mathrm{O}$. Here, we found that our plots displayed a semicircle in the highfrequency region and an inclined spur in the low-frequency region, in line with both literature precedent for RfA1 ${ }^{16}$ and expectations for ion-conducting materials contacted by blocking electrodes. ${ }^{33-37}$ Importantly, we also noted a clear kinetic isotope effect for the RfA2 films, with a decrease of $\sim 40 \%$ in their average conductivity upon moving from $\mathrm{H}_{2} \mathrm{O}$ to $\mathrm{D}_{2} \mathrm{O}$ vapor. Together, our observations provided additional compelling evidence that RfA2 was a proton-conducting material.

In conclusion, we have demonstrated an improved methodology for the production of reflectins in quantities sufficient for materials applications, and our findings constitute a significant advance for several reasons. First, our straightforward protocol omits a challenging chromatography step and enables the expression and purification of potentially difficult-to-handle reflectins in high yield, as demonstrated for the RfA2 "model" system. The presented strategy should be broadly applicable for the production of arbitrary reflectin variants (and perhaps even unrelated proteins) that are sequestered within inclusion bodies (for example, we have included the gel electrophoresis data for RfA1 produced via our protocol in ESI Fig. S7 $\dagger$ ). Furthermore, our methodology has enabled the exploration of RfA2's electrical functionality for the first time, adding to the limited body of knowledge currently available for reflectins' materials properties. Based on the sequence similarity between various reflectins and the demonstrated electrical functionality of both RfA1 and RfA2, we cautiously postulate that protonic conductivity may be a general property of these proteins (although our hypothesis certainly warrants additional investigation). Finally, due to its relatively short amino acid sequence (when compared to RfA1), RfA2 may hold value as a platform for establishing relationships between structure and in vitro function for the reflectin family of proteins. Altogether, our observations highlight the potential of reflectins as functional materials and may afford new opportunities for the study of their multi-faceted properties.

\section{Acknowledgements}

We thank Professor Albert Yee's Laboratory for the use of their optical microscope. We are grateful to the Air Force Office of Scientific Research (FA9550-14-1-0144) for its financial support. W. G. W. IV acknowledges the National Science Foundation Postdoctoral Research Fellowship in Biology (DBI1306188) for its financial support.

\section{References}

1 N. J. Abbott, R. Williamson and L. Maddock, in Cephalopod Neurobiology: Neuroscience Studies in Squid, Octopus, and Cuttlefish, Oxford University Press, Oxford, 1995.

2 L. Borrelli and G. Fiorito, in Learning and Memory: A Comprehensive Reference, ed. J. H. Byrne, Academic Press, Oxford, 2008, ch. 1.31, pp. 605-627.

3 R. T. Hanlon and J. B. Messenger, in Cephalopod Behaviour, Cambridge University Press, United Kingdom, 1998.

4 L. M. Mäthger, E. J. Denton, N. J. Marshall and R. T. Hanlon, J. R. Soc., Interface, 2009, 6, 149-163.

5 R. T. Hanlon, C.-C. Chiao, L. M. Mäthger, K. C. Buresch, A. Barbosa, J. J. Allen, L. Siemann and C. Chubb, in Animal Camouflage: Mechanisms and Functions, ed. M. Stevens and S. Merilaita, Cambridge University Press, Cambridge, 2011, ch. 9, pp. 145-163.

6 G. Kaufman and C. Orlt, Kings of Camouflage, Producers. Cuttlefish: The Brainy Bunch" [Television Broadcast], Public Broadcasting Service (PBS), 2011.

7 L. Phan, W. G. Walkup IV, D. D. Ordinario, E. Karshalev, J.-M. Jocson, A. M. Burke and A. A. Gorodetsky, Adv. Mater., 2013, 25, 5621-5625.

8 L. Phan, D. D. Ordinario, E. Karshalev, W. G. Walkup IV, M. Shenk and A. A. Gorodetsky, J. Mater. Chem. C, 2015, 3, 6493-6498.

9 R. M. Kramer, W. J. Crookes-Goodson and R. R. Naik, Nat. Mater., 2007, 6, 533-538.

10 G. Qin, P. B. Dennis, Y. Zhang, X. Hu, J. E. Bressner, Z. Sun, W. J. Crookes-Goodson, R. R. Naik, F. G. Omenetto and D. L. Kaplan, J. Polym. Sci., Part B: Polym. Phys., 2013, 51, 254-264.

11 J. Jin, V. Reese, R. Coler, D. Carter and M. Rolandi, Adv. Healthcare Mater., 2014, 3, 349-353. 
12 P. Hassanzadeh, M. Kharaziha, M. Nikkhah, S. Shin, J. Jin, S. He, W. Sun, C. Zhong, M. Dokmeci, A. Khademhosseini and M. Rolandi, J. Mater. Chem. B, 2013, 1, 4217-4224.

13 A. Cooper, C. Zhong, Y. Kinoshita, R. Morrison, M. Rolandi and M. Zhang, J. Mater. Chem., 2012, 22, 3105-3109.

14 L. Phan, R. Kautz, J. Arulmoli, I. H. Kim, D. T. T. Le, M. A. Shenk, M. M. Pathak, L. A. Flanagan, F. Tombola and A. A. Gorodetsky, ACS Appl. Mater. Interfaces, 2016, 8, 278-284.

15 Y. J. Kim, W. Wu, S. E. Chun, J. Whitacre and C. J. Bettinger, Proc. Natl. Acad. Sci. U. S. A., 2013, 110, 20912-20917.

16 D. D. Ordinario, L. Phan, W. G. Walkup IV, J.-M. Jocson, E. Karshalev, N. Hüsken and A. A. Gorodetsky, Nat. Chem., 2014, 6, 596-602.

17 D. D. Ordinario, L. Phan, J.-M. Jocson, T. Nguyen and A. A. Gorodetsky, APL Mater., 2015, 3, 014907.

18 D. D. Ordinario, L. Phan, Y. Van Dyke, T. Nguyen, A. G. Smith, M. Nguyen, N. M. Mofid, M. K. Dao and A. A. Gorodetsky, Chem. Mater., DOI: 10.1021/ acs.chemmater.6b00336.

19 C. Zhong, Y. Deng, A. F. Roudsari, A. Kapetanovic, M. P. Anantram and M. Rolandi, Nat. Commun., 2011, 2, 476.

20 Y. Deng, E. Josberger, J. Jin, A. F. Rousdari, B. A. Helms, C. Zhong, M. P. Anantram and M. Rolandi, Sci. Rep., 2013, 3, 2481.

21 W. J. Crookes, L. L. Ding, Q. L. Huang, J. R. Kimbell, J. Horwitz and M. J. McFall-Ngai, Science, 2004, 303, 235-238.

22 A. R. Tao, D. G. DeMartini, M. Izumi, A. M. Sweeney, A. L. Holt and D. E. Morse, Biomaterials, 2010, 31, 793-801.

23 M. Izumi, A. M. Sweeney, D. G. DeMartini, J. C. Weaver, M. L. Powers, A. R. Tao, T. V. Silvas, R. M. Kramer, W. J. Crookes-Goodson, L. M. Mäthger, R. R. Naik, R. T. Hanlon and D. E. Morse, J. R. Soc., Interface, 2010, 7, 549-560.
24 R. Levenson, C. Bracken, N. Bush and D. E. Morse, J. Biol. Chem., 2016, 291, 4058-4068.

25 D. G. DeMartini, D. V. Krogstad and D. E. Morse, Proc. Natl. Acad. Sci. U. S. A., 2013, 110, 2552-2556.

26 D. G. DeMartini, M. Izumi, A. T. Weaver, E. Pandolfi and D. E. Morse, J. Biol. Chem., 2015, 290, 15238-15249.

27 D. G. DeMartini, A. Ghoshal, E. Pandolfi, A. T. Weaver, M. Baum and D. E. Morse, J. Exp. Biol., 2013, 216, 3733-3741. 28 L. Mäthger, S. L. Senft, M. Gao, S. Karaveli, G. R. R. Bell, R. Zia, A. M. Kuzirian, P. B. Dennis, W. J. CrookesGoodson, R. R. Naik, G. W. Kattawar and R. T. Hanlon, Adv. Funct. Mater., 2013, 23, 3980-3989.

29 L. F. Deravi, A. P. Magyar, S. P. Sheehy, G. R. R. Bell, L. M. Mäthger, S. L. Senft, T. J. Wardill, W. S. Lane, A. M. Kuzirian, R. T. Hanlon, E. L. Hu and K. K. Parker, J. R. Soc., Interface, 2014, 11, 20130942.

30 J. B. Messenger, Biol. Rev. Cambridge Philos. Soc., 2001, 76, 473-528.

31 J. L. Weiss, N. A. Evans, T. Ahmed, J. D. J. Wrigley, S. Khan, C. Wright, J. N. Keen, A. Holzenburg and J. B. C. Findlay, Biochim. Biophys. Acta, Biomembr., 2005, 1668, 164-174.

32 H. Morgan, R. Pethig and G. T. Stevens, J. Phys. E: Sci. Instrum., 1986, 19, 80-82.

33 E. Barsoukov and J. R. Macdonald, in Impedance Spectroscopy: Theory, Experiment and Applications, Wiley, 2nd edn, 2005.

34 X.-Z. Yuan, C. Song, H. Wang and J. Zhang, in Electrochemical Impedance Spectroscopy in PEM Fuel Cells: Fundamentals and Applications, Springer, 2012.

35 R. A. Huggins, Ionics, 2002, 8, 300-313.

36 Z. Xie, C. Song, B. Andreaus, T. Navessin, Z. Shi, J. Zhang and S. Holdcroft, J. Electrochem. Soc., 2006, 153, 173-178.

37 T. Soboleva, Z. Xie, Z. Shi, E. Tsang, T. Navessin and S. Holdcroft, J. Electroanal. Chem., 2008, 622, 145-152. 